\title{
Article \\ Design and Stability Analysis of a Wall-Climbing Robot Using Propulsive Force of Propeller
}

\author{
Peng Liang, Xueshan Gao *, Qingfang Zhang, Rui Gao, Mingkang Li, Yuxin Xu and Wei Zhu \\ School of Mechatronical Engineering, Beijing Institute of Technology, Beijing 100081, China; \\ 3120170095@bit.edu.cn (P.L.); 3120195161@bit.edu.cn (Q.Z.); 3120170156@bit.edu.cn (R.G.); \\ 3120200201@bit.edu.cn (M.L.); xuyuxin@bit.edu.cn (Y.X.); wei.zhu@bit.edu.cn (W.Z.) \\ * Correspondence: xueshan.gao@bit.edu.cn; Tel.: +86-137-1890-8152
}

Citation: Liang, P.; Gao, X.; Zhang, Q.; Gao, R.; Li, M.; Xu, Y.; Zhu, W. Design and Stability Analysis of a Wall-Climbing Robot Using Propulsive Force of Propeller. Symmetry 2021, 13, 37. https:// doi.org/10.3390/sym13010037

Received: 18 November 2020 Accepted: 24 December 2020 Published: 29 December 2020

Publisher's Note: MDPI stays neutral with regard to jurisdictional claims in published maps and institutional affiliations.

Copyright: () 2020 by the authors. Licensee MDPI, Basel, Switzerland. This article is an open access article distributed under the terms and conditions of the Creative Commons Attribution (CC BY) license (https: / / creativecommons.org/ licenses/by/4.0/).

\begin{abstract}
This article introduces a wall-climbing robot that uses the reverse thrust of the propeller as the adsorption force. The robot is symmetrically distributed in structure and the adsorption force is symmetrically distributed before and after so that it can adapt to the surface of a variety of different media materials and achieve stable adsorption and movement of a variety of wall surfaces. The robot mainly uses the reverse thrust of the aircraft propeller as the adsorption force to achieve wall adsorption. The robot relies on four wheels to move forward. The forward power mainly comes from the combined action of the propeller reverse thrust component and the front wheel driving force. During the movement of the robot, the steering is realized by the front wheel differential control. In this paper, we design the structure of a dual-propeller dynamic adsorption wall mobile robot, plan the movement process of the robot from the ground to the wall, analyze the stable adsorption conditions of the robot wall, and carry out the robot's motion performance and adaptability test under different ground/wall environments to verify that the robot is stable and feasible.
\end{abstract}

Keywords: wall-climbing robot; propeller; adhesion system; wheel

\section{Introduction}

In the last few years, there has been a growing interest in climbing robots for several civilian and industrial fields. These robots can replace humans in some tasks, such as bridge pier inspection, glass exterior wall cleaning, anti-terrorism investigation, ship welding cleaning, and fire dangerous goods testing [1].

According to the adsorption method, the robot can be divided into vacuum negative pressure, magnetic adsorption, bionic, and reverse thrust. According to the movement mode, it can be divided into wheel, crawler, foot, and wheel-foot compound movement. Vacuum negative pressure robots [2-4] are mostly used on walls with relatively smooth walls and are widely used for cleaning glass walls, but the adsorption surface is prone to gas leakage, and the wall surface has high requirements for flatness and almost no obstacle crossing ability. Magnetic adsorption wall mobile robots [5-9] are mostly used for the inspection of the inner and outer surfaces of large metal utensils. They are only suitable for the walls of magnetically conductive materials, which have poor wall adaptability. Bionic wall mobile robots are based on the use of bionics to make adhesive materials and attach them to the wall contact structure to adhere to the robot [10-14]; biomimetic nature animal foot characteristics for paste or hook [15-19]. This adhesive material is not self-cleaning, the driving control is complicated with fuzzy hook. In recent years, many scholars and research institutions have carried out a lot of research on anti-thrust wall adsorption robots [20-23]. Alkalla Mohamed G and others have successively developed EJBot I and EJBot II [24,25], which can be applied to a variety of vertical wall robots. Shin, Jae-Uk and others used a multi-axis rotorcraft to turn around $90^{\circ}$ and attach it to the wall after taking off from the ground [26-28]. During the attaching movement, the robot body control is more complicated. 
This article uses dual propellers as the reverse thrust power unit, and the front wheel differential steering power unit. Design and plan the robot movement from the ground to the wall, analyze the stable adsorption conditions of the robot wall, and carry out the robot's motion performance and adaptability test under different ground/wall environments. According to the reverse thrust of the propeller, the robot makes the robot move forward, backward, and cling to the ground/wall surface stably. The robot is not affected by the contact medium, the shape of the contact surface, whether the contact surface is smooth, and the unevenness of convexity and concave. The robot has a simple structure and control, easy operation, and excellent motion stability.

\section{Robot Design}

\subsection{Robot Structure Design}

The robot is composed of a front and rear platform frame, a front wheel drive system, a rear wheel driven system, two rotor power drive unit, a hinge connection unit, a control module, and a communication module. The overall model of the robot is shown in Figure 1. The rotor power is driven by the paddles installed on the brushless motor, and the brushless motor is installed on the connected fixed frame. The angle of the rotor is adjusted by the steering gear, and the rotor is fixed on the front and back moving platform, respectively. They are what provide the necessary thrust to hold and maneuver ascension while it's on the wall and while it's on the ground. This project uses two 10 inch propellers because they are smaller and lighter while still providing sufficient thrust. This size propeller also allows for smaller rotating mounts, which ultimately results in a small chassis. The brushless motors are $1200 \mathrm{KV}$ and they provide sufficient torque to operate the paddle. In order to avoid the influence of aerodynamic disturbance on the paddle, the front paddle rotates clockwise and the rear paddle rotates counterclockwise. Both the front and rear paddles produce reverse thrust to make the robot stick to the wall. The front wheels are driven by a DC (Direct Current) drive motor to move forward, backwards, and turn. The front wheel changes direction under the action of the differential speed of the DC drive motor, the front wheel platform frame drives the rotating hinge to steer and the overall mobile platform steering. In order to increase the friction with the contact surface, soft rubber tires are installed on the surface of the wheel body.

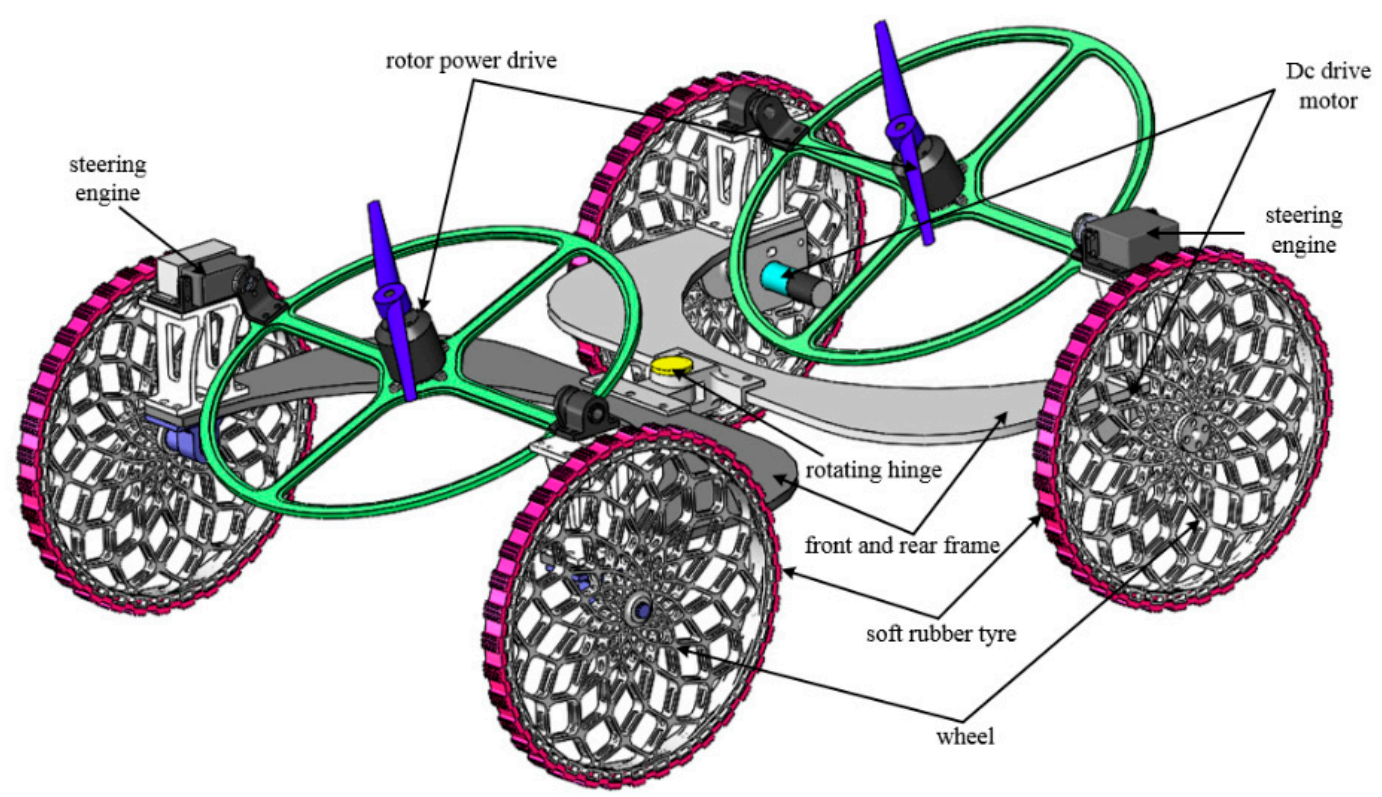

Figure 1. Overall diagram of multi-mode wall mobile robot. 
In order to control the overall weight of the robot, the body of the robot mostly uses 3D printed parts, and the platform frame is made of a composite of carbon fiber and honeycomb material. The structure parameters of the robot body are shown in Table 1 .

Table 1. Robot structure parameters.

\begin{tabular}{ccc}
\hline Name & Parameter Values & Unit \\
\hline Body size $\mathrm{L} \times \mathrm{W} \times \mathrm{H}$ & $600 \times 400 \times 200$ & $\mathrm{~mm}$ \\
Body total weight & 2.5 & $\mathrm{Kg}$ \\
Brushless DC motor output power & 400 & $\mathrm{~W}$ \\
(max) & 1200 & $\mathrm{KV}$ \\
Brushless DC Motor & TD-8120MG & $\mathrm{Kg} \cdot \mathrm{cm}$ \\
Micro servo & 22.8 & $\mathrm{~V}$ \\
Micro servo torque & 14.8 & $\mathrm{~V}$ \\
Battery voltage (4S) & 7.4 & $\mathrm{~mm}$ \\
Battery voltage (2S) & $254 \times 114$ & $\mathrm{~N}$ \\
Diameter $\times$ pitch & 25 & $\mathrm{~N}$ \\
Propeller lift (max) & 35.3 & \\
Adsorbability power (max)
\end{tabular}

\subsection{Robot Motion Design Planning}

The propeller reverse thrust robot uses the brushless motor to generate the reverse thrust to make the robot obtain the force of adsorption on the wall. By adjusting the change of the tilt angle of the front and rear rotors, the robot produces different motion effects. The robot movement process mainly includes ground/wall moving and turning, ground climbing to the wall, wall-to-wall, and wall-to-ground motion modes. During the movement of the ground/wall, the front drive system of the robot mobile platform drives the mobile platform and the driven system to move forward, backward, and turn.

The robot motion plan is shown in Figure 2. Figure $2 \mathrm{a}$ is a schematic diagram of the robot's turning, which is turned by the front wheel drive motor differential speed. Figure $2 b$ is an example of the robot moving from the ground to the vertical wall. In general, change the tilt angle of the front and rear rotors to adjust the robot's movement posture, and then the moving process of the mobile robot. When the robot approaches the wall from the ground, the front rotor power mechanism rotates $180^{\circ}$ to generate an upward pulling force perpendicular to the robot's forward direction. The front wheel mechanism can be raised, and under the action of the forward thrust of the rear rotor, the robot transitions from the ground to the wall. When the rear wheel is attached to the wall, the front propeller changes its angle, and the lift is transformed into the thrust of the suction wall. Under the combined action of the front and rear propellers, the robot sticks and moves on the wall.

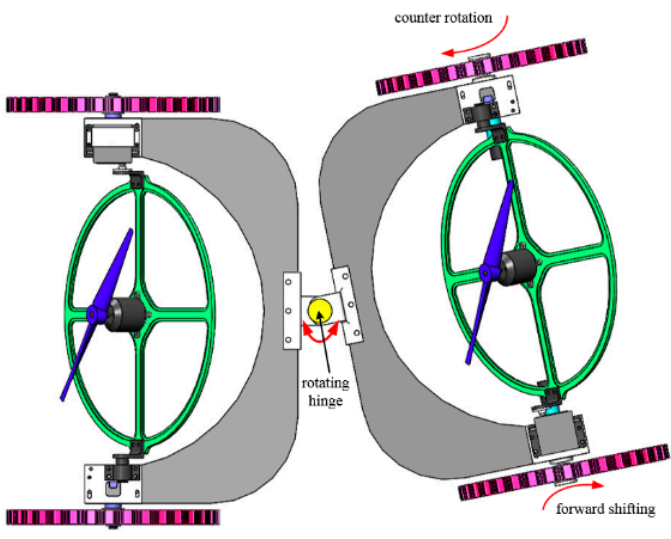

(a) robot turning: differential steering

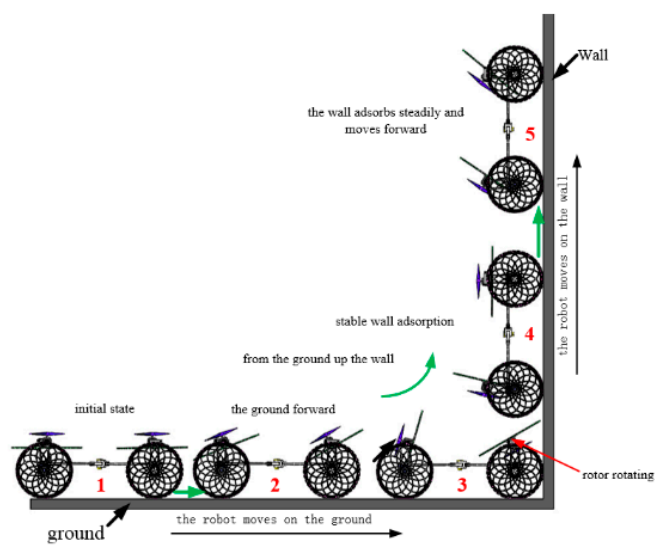

(b) robot forward, upper wall and wall movement

Figure 2. Example of transfer sequence between walls. 
In order to plan the robot motion process, it is usually necessary to obtain the robot motion state, which must be sensed and planned by some sensors attached to the robot itself.

\subsection{Propeller Power Test}

In order to get the actual reverse thrust of the rotor power system, it is necessary to test the rotor power. The rotor reverse thrust is generated by the controller to control the PWM (pulse width modulation), and the electronic governor adjusts the motor speed according to the pulse width, and the paddles are installed on the motor output shaft to directly generate the reverse thrust. Ignoring the resistance and lateral moment of the propeller, a single power unit produces mainly reverse thrust $F$ and torque $Q$. Both reverse thrust and torque are proportional to the square of the speed [29].

$$
\begin{aligned}
& F=\rho A C_{F} R^{2} \omega^{2}=k \omega^{2} \\
& Q=\rho A C_{Q} R^{2} \omega^{2}=d \omega^{2}
\end{aligned}
$$

where: $C_{F}, C_{Q}$ are the pull coefficient and torque coefficient of the rotor; $\rho$ is the air density; $A=\pi R^{2}$ is the area of the propeller; $\rho, A, C_{F}, C_{Q}, R$ are the lift coefficients; $k$ is all fixed values; $d$ is the torque numbers.

It can be seen from the above formula that the reverse thrust of the rotor is proportional to the square of the motor speed, and the square of the speed is proportional to the current. Therefore, it is estimated that the propeller output reverse thrust is also proportional to the controller output current. Rotor reverse thrust test experimental device, including battery, digital display tensile tester, brushless motor, paddle, electronic governor, receiver, remote control, etc., as shown in Figure 3.

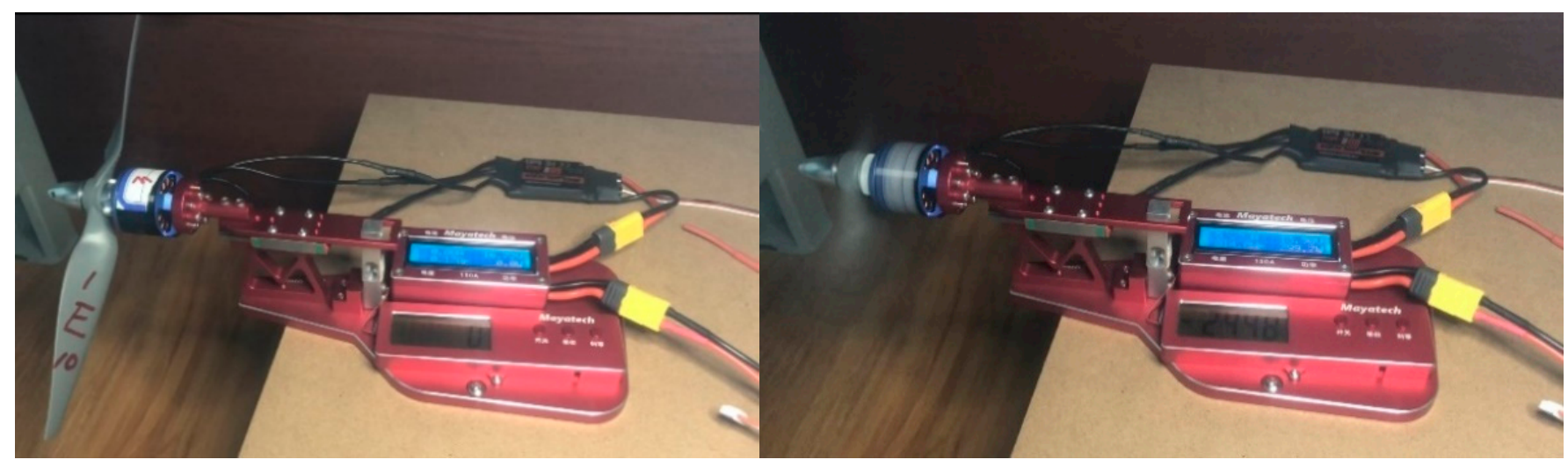

Figure 3. Experimental device for reverse thrust test of propeller motor.

Figure 4 shows the propeller motor reverse thrust test curve. Both curves are the reverse thrust curve obtained by the reverse installation of the 10 inches paddle. The purple data curve is the data measured under the condition that the paddle is mounted backwards, and the motor rotates clockwise. The blue data curve is the measured data curve when the paddle is reversely installed, and the motor rotates counterclockwise. It can be clearly seen from the figure that the reverse thrust increases with the increase of the current value. When the current value is $50 \mathrm{~A}$, the reverse thrust is the largest $25 \mathrm{~N}$. Changing the PWM value again makes it impossible to adjust the motor speed, and the power output of the power system reaches its peak value. 


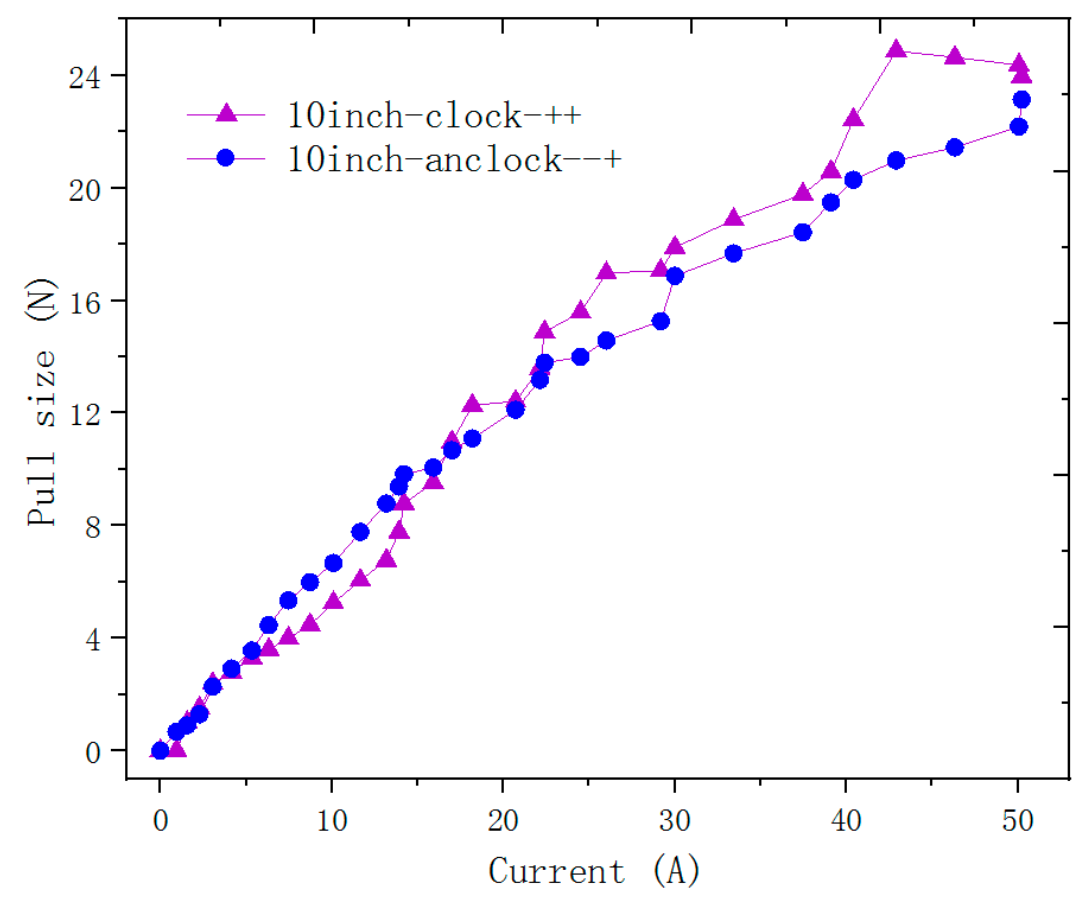

Figure 4. Propellor motor reverse thrust pull curve.

\subsection{Force Analysis of the Robot Movement Process}

The reverse thrust wall-climbing robot is in an uphill state, which is an action posture that the robot must experience during its movement. That is, when the robot is traveling, the front wheel is lifted under the combined action of the rotor flip and the rear wheel reverse thrust. The state attached to the slope, the force of the robot in this state is shown in Figure 5.

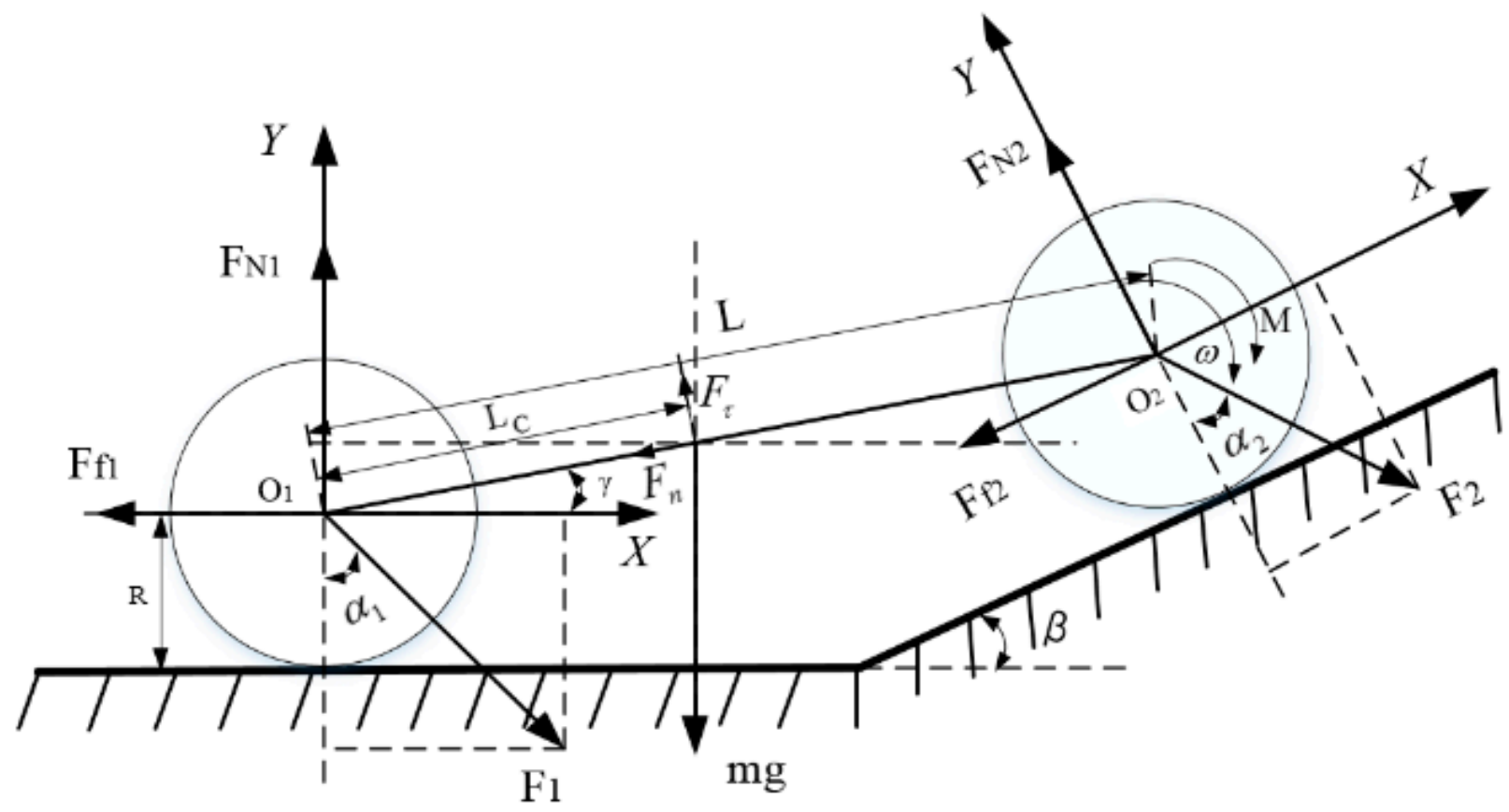

Figure 5. Force diagram when the robot is uphill. 
According to the Newton-Euler equation and the equilibrium condition for the system of particles [30], we can get:

$$
\left\{\begin{array}{l}
\sum F_{i}^{e}+\sum F_{g i}=0 \\
\sum m_{j}\left(F_{i}^{e}\right)+\sum m_{j}\left(F_{g i}\right)=0
\end{array}\right.
$$

Among them:

$F_{i}^{e}-i$ point external force;

$F_{g i}-i$ point inertial force;

$\sum m_{j}\left(F_{i}^{e}\right)$ - the moment of the mass $i$ point relative to the $j$ axis;

$\sum m_{j}\left(F_{g i}\right)$ - the moment of inertia of the $i$ particle relative to the $j$ axis;

As shown in the figure above, the coordinate system $\mathrm{XO}_{1} Y$ is established, the origin of the coordinates is the center point of the driving wheel $O_{1}$, the horizontal line is the $X$ axis, and the normal line of the horizontal line passing through the origin is the $Y$ axis. The establishment of the uphill dynamic model of the reverse thrust wall-climbing robot can be obtained:

$$
\left\{\begin{array}{l}
F_{1} \sin \alpha_{1}+F_{2} \sin \left(\alpha_{2}+\beta\right)-F_{f 1}-F_{f 2} \cos \beta-F_{N 2} \sin \beta-F_{\tau} \sin \gamma-F_{n} \cos \gamma=0 \\
F_{N 1}+F_{N 2} \cos \beta-F_{1} \cos \alpha_{1}-F_{f 2} \sin \beta-F_{2} \cos \left(\alpha_{2}+\beta\right)-m g+F_{\tau} \cos \gamma-F_{n} \sin \gamma=0 \\
F_{N 2} L+F_{\tau} L_{C}-F_{2} L \sin \left(\alpha_{2}+\beta-\gamma\right)-F_{f 2} L \sin (\beta-\gamma)-m g L_{C} \cos \gamma=0 \\
-F_{\tau}\left(L-L_{C}\right)-F_{N 1} L \cos \gamma-F_{f 1} L \sin \gamma-M+F_{1} L \cos \left(\alpha_{2}-\gamma\right)+m g\left(L-L_{C}\right) \cos \gamma=0
\end{array}\right.
$$

From this structure, it can be seen that the rotor generates reverse thrust to act on the front and rear platform frames of the robot, so that it can be stably adsorbed on the wall. The normal force of the reverse thrust causes friction between the front and rear wheels and the wall, tangentially forces overcome gravity and move up the wall. The robot's gravity moves downward vertically. The force analysis diagram of the robot adsorbed on the wall is shown in Figure 6.
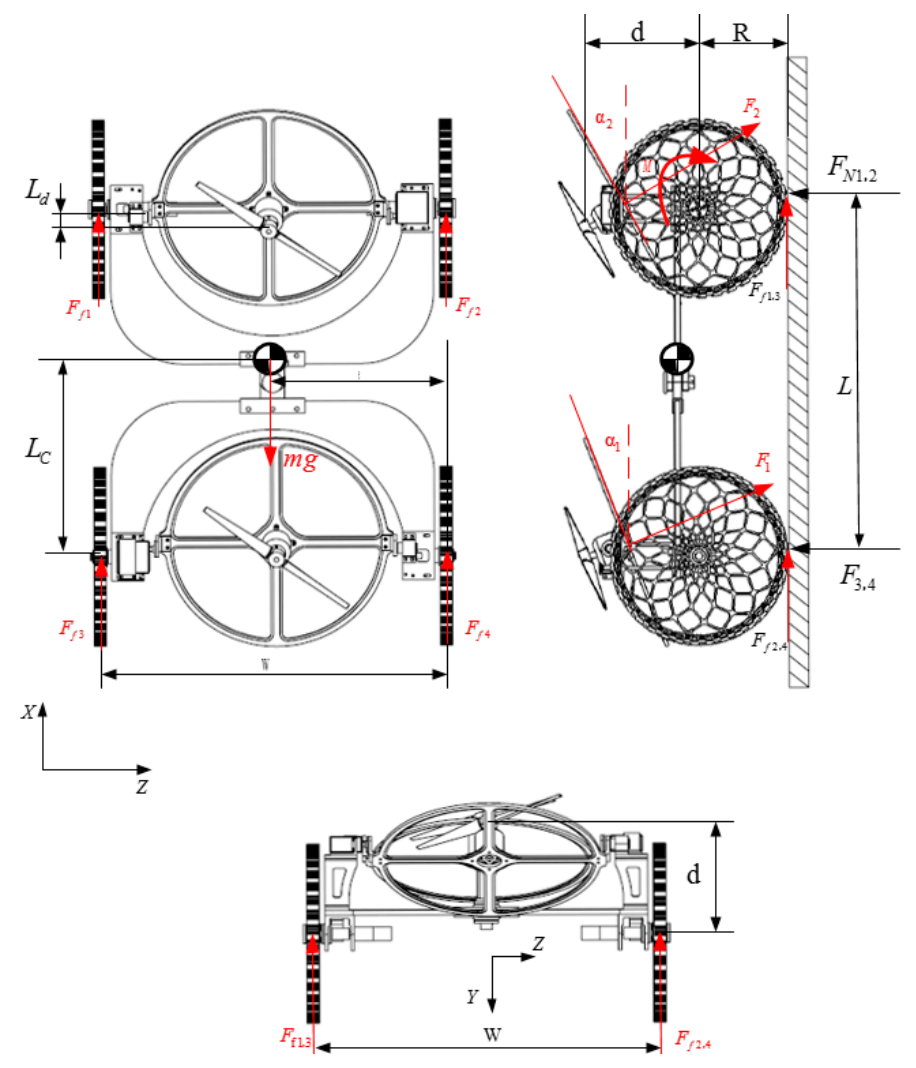

Figure 6. The force analysis diagram of the robot adsorbed on the wall. 
$F_{N i}$ is the normal force acting on the wheel $i$ respectively, $F_{f i}$ is the frictional force acting between the $i$ wheel and the contact wall, $F_{2}$ and $F_{1}$ are the reverse thrust generated by the front and rear rotors, the direction of the force is perpendicular to the blade mounting fixing plate. $\alpha_{2}$ and $\alpha_{1}$ are the tilt angle of front and rear rotors, that is, the elevation angle of the rotor and the robot; $\mathrm{R}$ is the wheel radius; $\mathrm{d}$ is the distance from the support frame to the axis of the blade along the $Z$ axis; $L_{d}$ is the distance from the axis of the wheel to the axis of the blade along the $X$ axis; $L_{c}$ is the distance from the axis of the rear wheel to the particle of the robot body along the $X$ axis; $L$ is the distance between the front and rear wheels; $\mathrm{W}$ is the distance between the left and right wheels; $\mathrm{e}$ is the distance between the right turn and the center of mass along the $\mathrm{Z}$ axis.

From the structural characteristics of the robot, it can be concluded that the instability of the robot is mainly caused by the slip of the contact wheel and the side of the robot on the wall. The condition that the robot does not produce slippage, the upward force of the robot should be greater than the downward force along the slope. According to the force balance condition, when the robot hovering stably on the wall, we can get:

$$
\begin{aligned}
& F_{N 1}+F_{N 2}+F_{N 3}+F_{N 4}=F_{1} \cos \alpha_{1}+F_{2} \cos \alpha_{2} \\
& F_{f 1}+F_{f 2}+F_{f 3}+F_{f 4}+F_{1} \sin \alpha_{1}+F_{2} \sin \alpha_{2}=m g \\
& -\left(F_{f 1}+F_{f 3}\right)(\mathrm{w}-e)+\left(F_{f 2}+F_{f 4}\right) e-F_{1}\left(\sqrt{\left(L_{\mathrm{c}}-L_{d}\right)^{2}+d^{2}} \cos \left(\arctan \left(\frac{d}{L_{c}-L_{d}}\right)+\alpha_{1}\right)\right) \\
& +F_{2}\left(\sqrt { ( L - L _ { \mathrm { c } } - L _ { d } ) ^ { 2 } + d ^ { 2 } } \operatorname { c o s } \left(\arccos \sqrt{\frac{\left(L-L_{\mathrm{c}}-L_{d}\right)^{2}+d^{2}+\left(L-L_{\mathrm{c}}\right)^{2}-\left(L_{d^{2}}+d^{2}\right)}{2 \sqrt{\left(L-L_{\mathrm{c}}-L_{d}\right)^{2}+d^{2}}\left(L-L_{\mathrm{c}}\right)}}\right.\right. \\
& \left.-\arccos \sqrt{\left.\frac{\left(L_{d}^{2}+d^{2}\right)+\left(L-L_{\mathcal{C}}\right)^{2}-\left(L-L_{\mathrm{c}}-L_{d}\right)^{2}}{2 \sqrt{L_{d^{2}+d^{2}}}\left(L-L_{\mathrm{c}}\right)}\right)}\right)=0 \\
& -\left(F_{N 1}+F_{N 2}\right)(w-e)+\left(F_{N 3}+F_{N 4}\right) e=0 \\
& -\left(F_{N 1}+F_{N 2}\right)\left(L-L_{c}\right)-F_{1}\left(\sqrt{\left(L_{c}-L_{d}\right)^{2}+d^{2}} \cos \left(\arctan \left(\frac{d}{L_{c}-L_{d}}\right)+\alpha_{1}\right)\right)+ \\
& F_{2}\left(\sqrt { ( L - L _ { \mathrm { c } } - L _ { d } ) ^ { 2 } + d ^ { 2 } } \operatorname { c o s } \left(\arccos \sqrt{\frac{\left(L-L_{\mathrm{c}}-L_{d}\right)^{2}+d^{2}+\left(L-L_{\mathrm{c}}\right)^{2}-\left(L_{\left.d^{2}+d^{2}\right)}\right.}{2 \sqrt{\left(L-L_{\mathrm{c}}-L_{d}\right)^{2}+d^{2}\left(L-L_{\mathrm{c}}\right)}}}\right.\right. \\
& \left.\left.-\arccos \sqrt{\frac{\left(L_{d}^{2}+d^{2}\right)+\left(L-L_{\mathcal{c}}\right)^{2}-\left(L-L_{\mathrm{c}}-L_{d}\right)^{2}}{2 \sqrt{L_{d}{ }^{2}+d^{2}}\left(L-L_{\mathrm{c}}\right)}}\right)\right)+\left(F_{N 3}+F_{N 4}\right) L_{c}=0
\end{aligned}
$$

The model is statistically indeterminate problem thus, the force displacement method for compatibility is used with considering the chassis is as a rigid body, thus, the last equation is as follows:

$$
\frac{F_{N 1}}{k_{w 1}}-\frac{F_{N 2}}{k_{w 2}}-\frac{F_{N 3}}{k_{w 3}}+\frac{F_{N 4}}{k_{w 4}}=0
$$

where, $K_{w i}$ is the stiffness of wheel $i$. The frictional force $F_{f i}$ should always be less than the normal force $F_{N i}$ multiplied by the static coefficient of friction $\mu_{s}$ as follows:

$$
F_{f i} \leq \mu_{s} F_{N i}(i=1, \cdots, 4)
$$

The relation between the wheels torques and the frictional forces as follows:

$$
M=F_{f i} \cdot R(i=1, \cdots, 4)
$$

\subsection{Robot Electrical System Design}

STM32 6-way Arduino is selected as the underlying embedded controller, and the internal measurement unit is IMU (Inertial Measurement Unit). It uses a combination of angular velocity meter and gyroscope sensor to construct a system detection unit to determine the angle and angular velocity of the robot. An Intertia Measurement Unit, which is a combination of accelerometer and gyro sensor, is used to construct the observer system in order to determine the robot angle and angular velocity. Two 60A BLDC ESC is used as a brushless motor driver, which can drive each brushless motor up to $60 \mathrm{~A}$ continuous driving current. Two servo motors are used to control the change of the inclination angle of the rotor, 
two quadcopter motors are used, they need more power to operate. This means a bigger battery is needed. For testing times and run times of around five minutes operating the motors at full speed as well as powering the processor, IMU, and servos, a 4 cell $4000 \mathrm{mAh}$ battery is used. The transmitter/receiver pair is used for long range control of the vehicle. It operates using a $2.4 \mathrm{GHz}$ frequency, which can allow for ranges up to $20 \mathrm{~m}$ of control. The receiver outputs a PWM signal to the on-board processor, which then determines the user's desired direction of travel. The electronics system is shown in Figure 7.

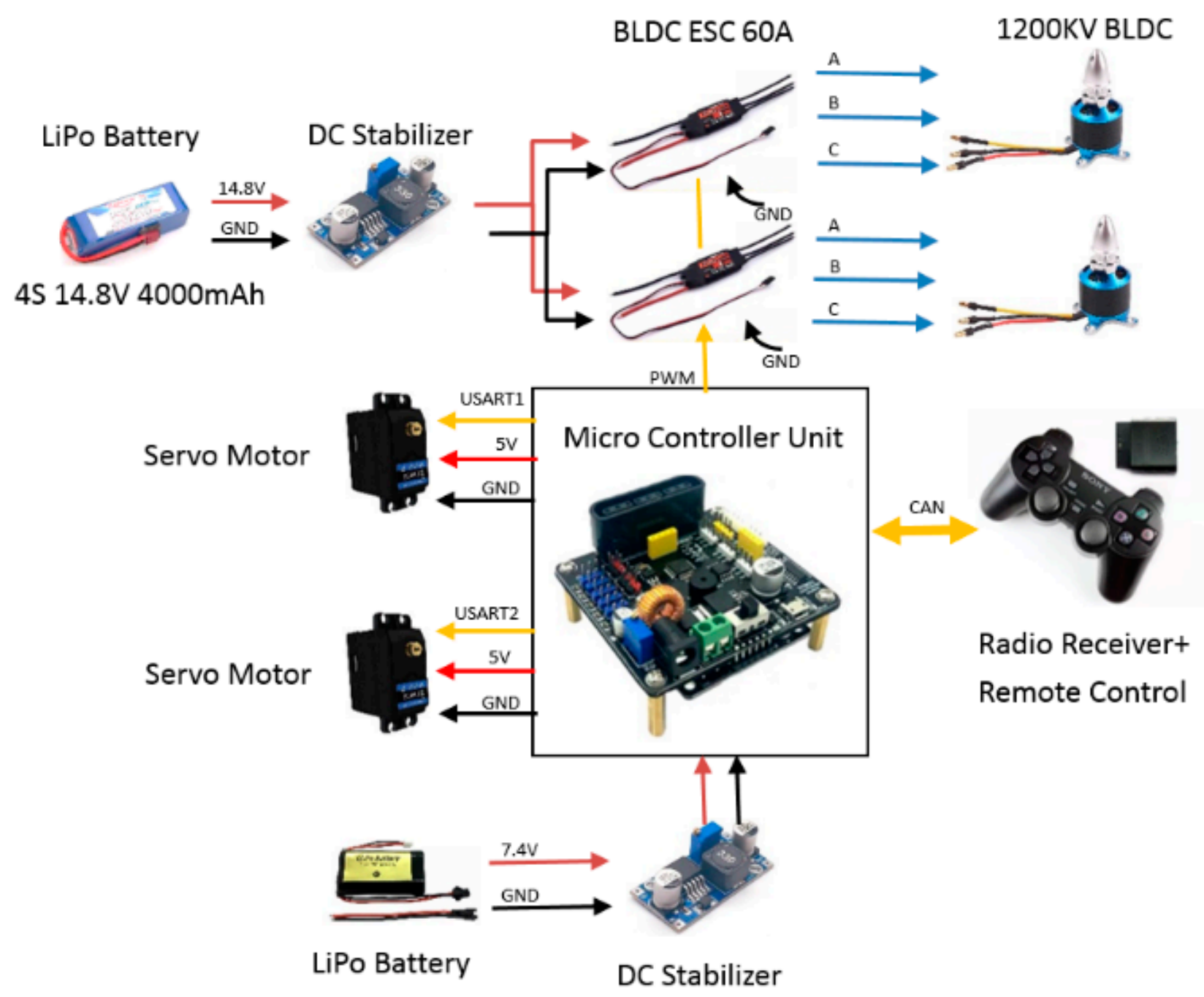

2S 7.4V 2200mAh

Figure 7. Electronics system.

\section{Experiment}

3.1. Traction Measurement Experiment

In order to obtain the maximum traction force of the robot under different tilt angles of the rotor, the horizontal tension value of the mobile platform of the robot in different gears and different rotor tilt angles is measured with a spring tension meter (considering safety and laboratory site restrictions, the maximum gear of the robot is only open during actual measurement to 6 gears, 10 gears are designed), as shown in Figure 8, the robot traction force measurement under different rotor tilt angles. 


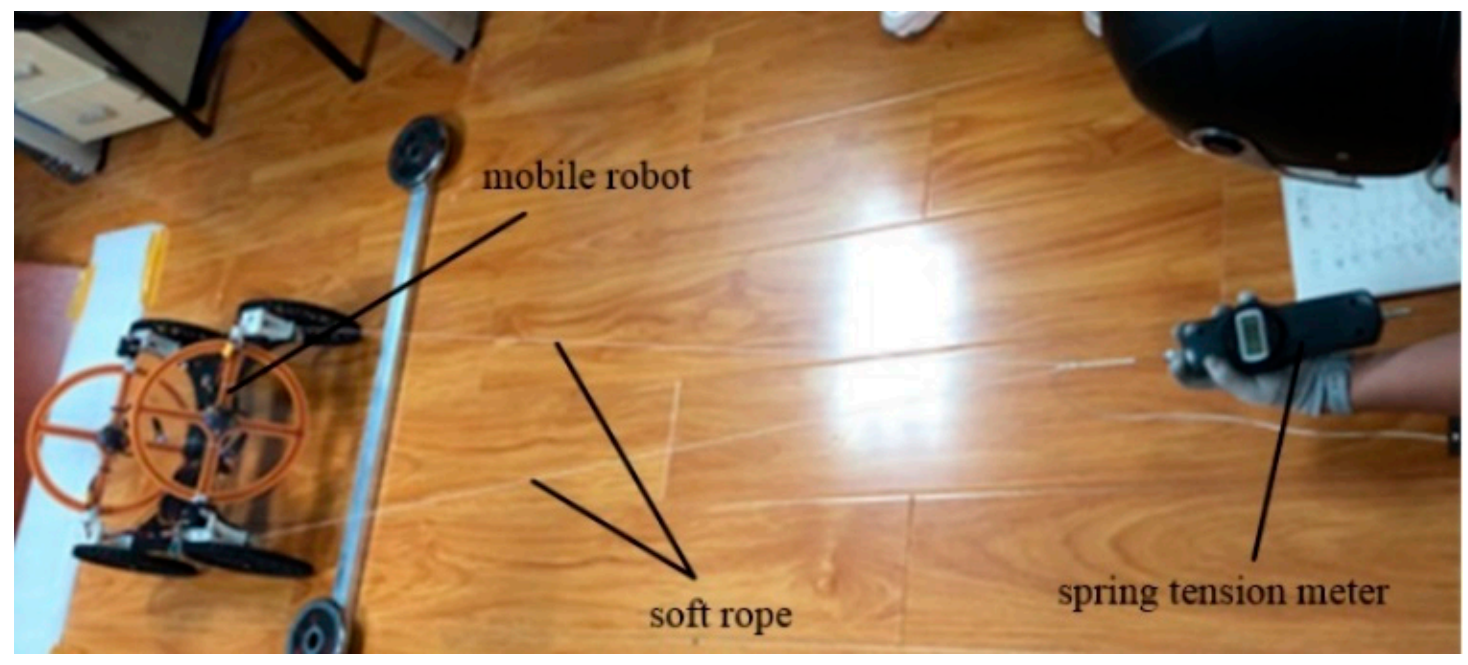

Figure 8. Robot traction measurement under different rotor tilt angles.

In order to improve the measurement accuracy, the measurement data of the change of the inclination angle under different gears are shown in Figure $9 \mathrm{a}$, and the measurement data of the change of the gear under different inclination angles are shown in Figure 9b. Figure 9 shows that as the gear increases, the reverse thrust continues to increase. When the rotor tilt angle is $60^{\circ}$, the robot obtains the maximum traction.

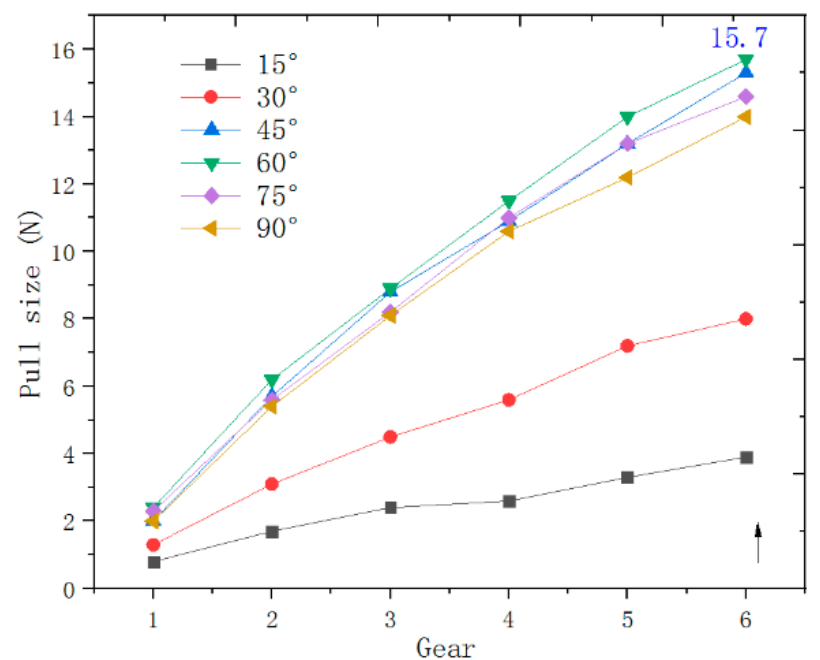

(a) Tilt angle changes in different gears

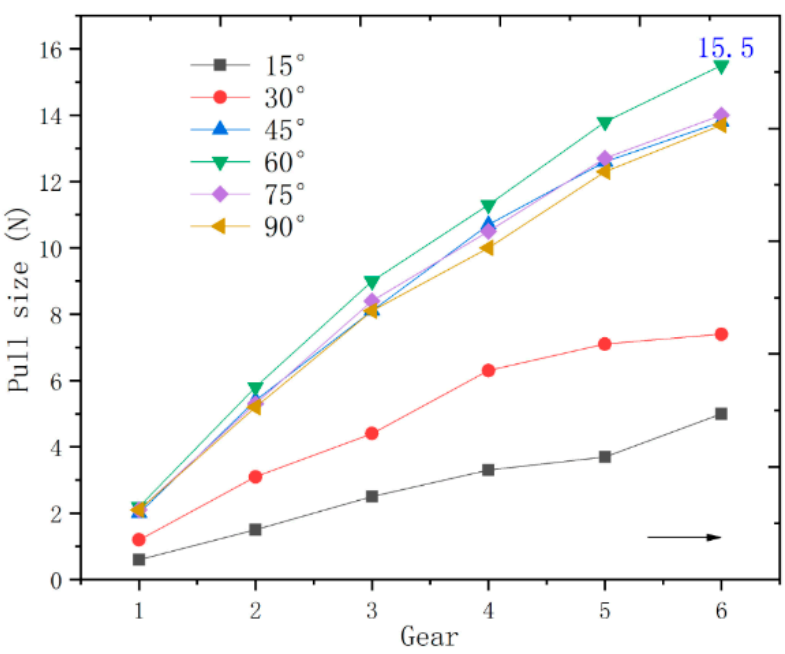

(b) Change of gear change at different inclination angles

Figure 9. Robot traction measurement data on the ground.

\subsection{Small Slope Measurement Experiment}

In order to verify the feasibility of robot motion and whether it can be stably adsorbed on the wall, the following experiments are specially designed for verification. As the robot moves from the ground to the wall at a small angle, the robot moves directly to the inclined surface at the initial speed of the ground. When the inclination angle of the bottom plate increases to a certain extent, the robot touches the bottom plate from the ground. The impact force can easily cause damage to the robot structure, so the small-angle slope ground-to-wall motion test is designed to $45^{\circ}$. As shown in Figure 10, describe the robot's movement from the ground to $20^{\circ}, 25^{\circ}$ and $45^{\circ}$ slopes. When the robot moves from the ground to a slope with a certain inclination angle, when the robot moves from the ground to a small slope, the rotor tilt angle is relatively large. You can climb it when you are young. 
As the angle of the inclined plane increases, a larger rotor inclination angle and blade rotation speed are required.

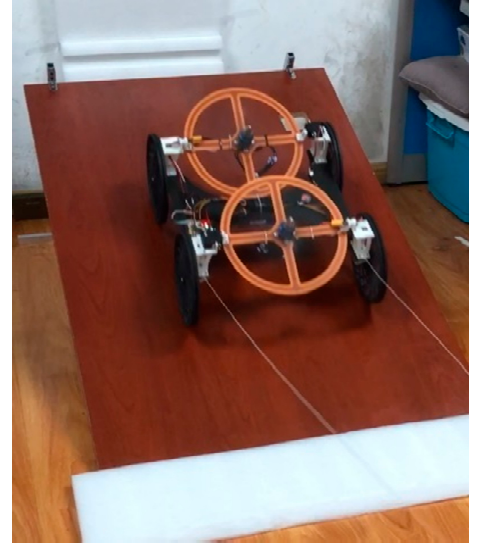

$20^{\circ}$ slope

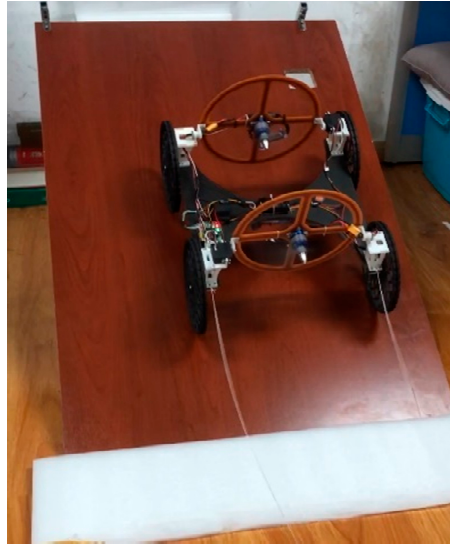

$25^{\circ}$ slope

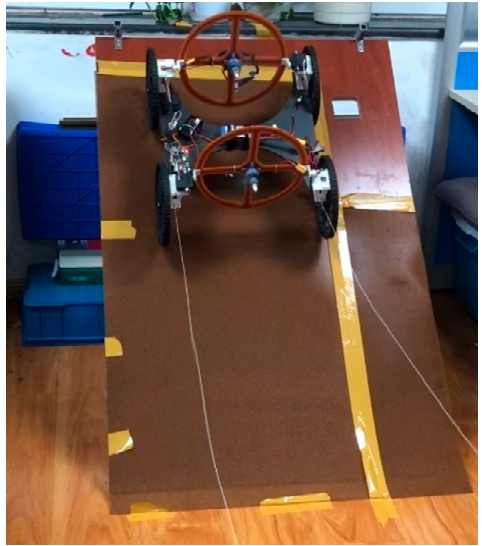

$45^{\circ}$ slope

Figure 10. Robot stable motion test under different slopes.

\subsection{Vertical Wall Measurement Experiment}

In order to measure that the robot can be stably attached to the vertical wall, the robot is hoisted on the vertical wall. To prevent the robot from rushing out of the wall, a certain length of soft rope is installed under the robot so that the robot can move within a certain range of the wall. The robot wall surface stable adsorption movement process is shown in Figure 11a-h. (a) Initial state; (b) Movement start; (c) Rotor tilt angle adjustment; (d) Rotor tilt angle adjusted to $60^{\circ}$, the upper end rope starts to relax; (e) The upper end rope is completely loose; (f) The robot starts to move upwards; ( $g$ ) The bottom limit rope is straightened, and the robot can be completely adsorbed on the wall; (h) The speed of the blade is reduced, and the robot falls.

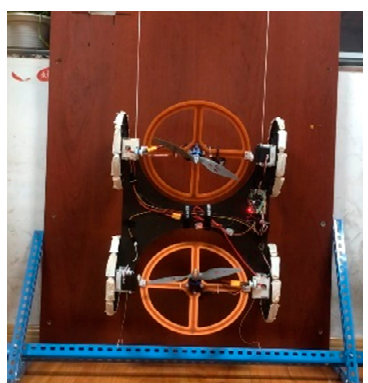

(a)

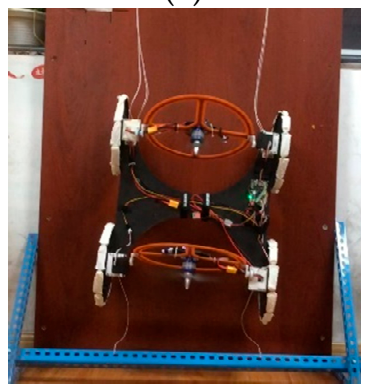

(e)

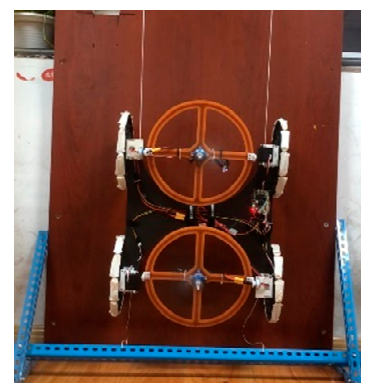

(b)

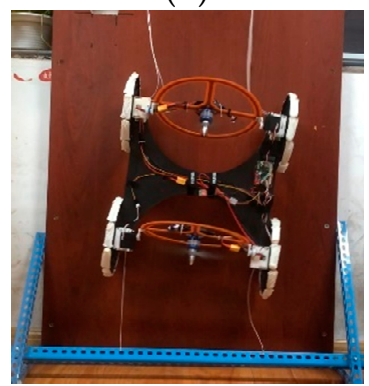

(f)

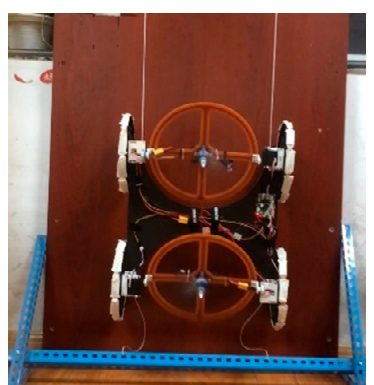

(c)

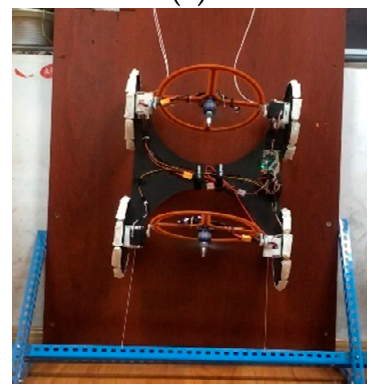

(g)

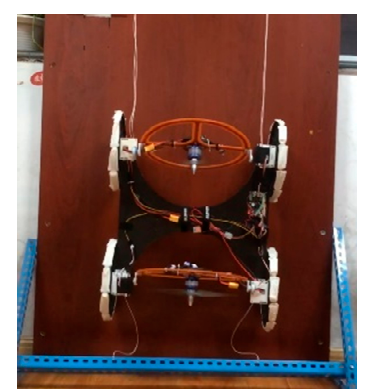

(d)

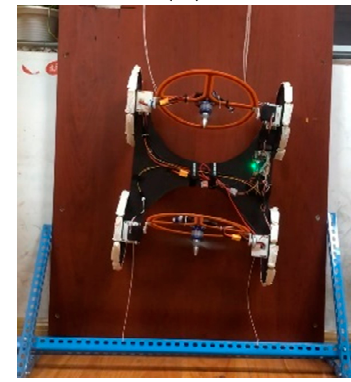

(h)

Figure 11. Stable adsorption movement process of the robot wall. 
During the test movement, the upper and lower traction ropes in Figure $11 \mathrm{~d}-\mathrm{f}$ are in an unstressed state, the maximum rotor speed of the robot is 7 gears, and the robot can stably adsorb on the vertical wall. The smooth progress of the experiment lays a good foundation for carrying out ground to wall compliant control of the robot in the later stage.

\section{Conclusions}

In this paper, a dual-propeller wall-climbing robot that can stably adsorb on a variety of different walls is designed. By analyzing the robot's movement from the ground to the wall and the robot's stable adsorption conditions on the wall, a dual-propeller-type wall mobile robot is produced. The robot climbing experiment under a small slope and the $90^{\circ}$ slope stable adsorption experiment verified the feasibility of the robot moving stably on the vertical wall.

Future work should aim to optimize the robot structure model, manufacture a frontwheel drive with a rotatable prototype for the front and rear wheel fixed brackets, carry out experimental research on the robot's forward, backward, and turn tests in a real terrain environment, and add ranging and gyroscope sensors to sense the robot's position and posture. By adjusting and correcting the robot posture, further steps can be taken to obtain the appropriate robot motion state and lay the theoretical and experimental accumulation for realizing the smooth transition from the ground to the wall of the robot.

Author Contributions: Investigation, R.G. and W.Z.; Methodology, P.L. and X.G.; Project administration, Y.X.; Software, Q.Z. and M.L. All authors have read and agreed to the published version of the manuscript.

Funding: National Key Research and Development Program of China (2019YFB1309600). Graduate Technological Innovation Project of Beijing Institute of Technology (2019CX20008).

Institutional Review Board Statement: Not applicable.

Informed Consent Statement: Not applicable.

Data Availability Statement: Data sharing not applicable.

Conflicts of Interest: The authors declare no conflict of interest.

\section{References}

1. Seo, T.W.; Jeon, Y.; Park, C. Survey on Glass and Facade-Cleaning Robots: Climbing Mechanisms, Cleaning Methods, and Applications. Int. J. Precis. Eng. Manuf. Green Technol. 2019, 6, 367-376. [CrossRef]

2. Li, J.; Xueshan, G.; Ningjun, F.; Kejie, L.; Zhihong, J.; Zhijian, J. Adsorption Performance of Sliding Wall-Climbing Robot. Chin. J. Mech. Eng. 2010, 23, 733-741. [CrossRef]

3. Viraj, M.A.; Muthugala, M.; Rajesh Elara, M.; Suresh, R. Design and Control of a Wall Cleaning Robot with Adhesion-Awareness. Symmetry 2020, 12, 1-18.

4. Qiang, Z.; Li, X. Experimental investigation on climbing robot using rotation-flow adsorption unit. Robot. Auton. Syst. 2018, 105, 112-120.

5. Fabien, T.; Wolfgang, F.; Gilles, C.; Roland, S.; Roland, M.; Francesco, M. Magnebike: A Magnetic Wheeled Robot with High Mobility for Inspecting Complex-Shaped Structures. J. Field Robot. 2009, 26, 453-476.

6. Tirthankar, B.; Ryan, S.; Fletcher, T.; Navinda, K.; Ross, D.; Brett, W.; James, B.; Karsten, H.; Alberto, E. Magneto: A Versatile Multi-Limbed Inspection Robot. In Proceedings of the 2018 IEEE/RSJ International Conference on Intelligent Robots and Systems (IROS), Madrid, Spain, 1-5 October 2018; pp. 2253-2260.

7. Feifei, Z.; Xuerong, S.; Zhanpeng, L.; Imran, M.; Yanan, W.; Kai, H. Influence of Processing Parameters on Coating Removal for High PressureWater Jet Technology Based on Wall-Climbing Robot. Appl. Sci. 2020, 10, 1-18.

8. David, P.; Dikai, L. An approach for real-time motion planning of an inchworm robot in complex steel bridge environments. Robotica 2017, 35, 1280-1309.

9. Xueshan, G.; Dianguo, X.; Yan, W.; Huanhuan, P.; Weimin, S. Multifunctional robot to maintain boiler water-cooling tubes. Robotica 2009, 27, 941-948.

10. Sangbae, K.; Spenko, M.; Trujillo, S. Smooth vertical surface climbing with directional adhesion. IEEE Trans. Robot. 2008, 24, 65-74. [CrossRef]

11. Giuk, L.; Hwang, K.; Kunchan, S.; Jongwon, K.; Metin, S.; Taewon, S. Series of Multilinked Caterpillar Track-type Climbing Robots. J. Field Robot. 2016, 33, 737-750. 
12. Xuan, W.; Rongchuan, W.; Tao, M.; Xiaojie, W.; You, L. A wall-climbing robot using gecko-inspired dry adhesives and underactuated modular connecting mechanism. Bioinspirat. Biomim. Bioreplicat. 2018, 10593, 1-11.

13. Yanheng, L.; HyunGyu, K.; TaeWon, S. AnyClimb: A New Wall-Climbing Robotic Platform for Various Curvatures. IEEE Asme Trans. Mechatron. 2016, 21, 1812-1821.

14. Dharmawan, A.; Xavier, P.; Hariri Hassan, H. Design, Modeling and Experimentation of a Bio-Inspired Miniature Climbing Robot with Bilayer Dry Adhesives. J. Mech. Robot. Trans. Asme 2019, 11, 1-9. [CrossRef]

15. Aksak, B.; Murphy, M.P.; Sitti, M. Gecko inspired micro-fibrillar adhesives for wall climbing robots on micro/nanoscale rough surfaces. In Proceedings of the 2008 IEEE International Conference on Robotics and Automation, Pasadena, CA, USA, 19-23 May 2008; pp. 3058-3063.

16. Sangbae, K.; Asbeck, A.T.; Cutkosky, M.R.; Provancher, W.R. SpinybotII: Climbing hard walls with compliant microspines. In Proceedings of the ICAR 12th International Conference on Advanced Robotics, Seattle, WA, USA, 18-20 July 2005 ; pp. 601-606.

17. Austin, M.P.; Brown, J.M.; Young, C.A.; Clark, J.E. Leg Design to Enable Dynamic Running and Climbing on BOBCAT. In Proceedings of the 2018 IEEE/RSJ International Conference on Intelligent Robots and Systems, Madrid, Spain, 1-5 October 2018; pp. 3799-3806.

18. Bruce, D.M.; Peter, R.R.; James, D.D.; Jonathan, E.C. Running up a wall: The role and challenges of dynamic climbing in enhancing multi-modal legged systems. Bioinspirat. Biomim. 2015, 10, 1-16.

19. Jason, M.B.; Max, P.A.; Bruce, D.M.; Jonathan, E.C. Evidence for multiple dynamic climbing gait families. Bioinspirat. Biomim. 2019, 14, 1-16.

20. Nishi, A.; Miyagi, H. A Wall-Climbing robot using propulsive force of propeller-mechanism and control-system in a strong wind. Int. J. Ser. C Dyn. Control Robot. Des. Manuf. 1994, 37, 172-178. [CrossRef]

21. Kanjanapan, S.; Pruttapon, M.; Konlayut, S. Design of a Double-propellers Wall-Climbing Robot. In Proceedings of the 2017 IEEE International Comference on Robotics and Biomimetics, Macau, China, 5-8 December 2017; pp. $239-245$.

22. Beardsley, P. VertiGo-A Wall—Climbing Robot Including Ground-Wall Transition; Disney Research Zurich. 2015. Available online: https: / la.disneyresearch.com/publication/vertigo/ (accessed on 17 November 2020).

23. Ioi, K.; Kito, T.; Tanaka, Y. Dynamic Simulator for Design and Control of a Wall-climbing Robot. In Proceedings of the 2019 IEEE/SICE International Symposium on System Integration, Paris, France, 14-16 January 2019; pp. 40-44.

24. Mohamed, G.A.; Mohamed, A.F.; Abdelfatah, M.M. Tele-operated propeller-type climbing robot for inspection of petrochemical vessels. Ind. Robot Int. J. Robot. Res. Appl. 2017, 44, 166-177.

25. Mohamed, G.A.; Mohamed, A.F.; Abdel-Fattah, M.; Shuji, H.; Hideyuki, S.; Takanobu, M. EJBot-II: An optimized skid-steering propeller-type climbing robot with transition mechanism. Adv. Robot. 2019, 33, 1042-1059.

26. Jae-Uk, S.; Donghoon, K.; Jong-Heon, K.; Hyun, M. Micro-aerial vehicle type wall-climbing robot mechanism for structural health monitoring, Sensors and Smart Structures Technologies for Civil. Mech. Aerosp. Syst. 2013, 8692, 1-7.

27. Myeong, W.; Myung, H. Development of a Wall-Climbing Drone Capable of Vertical Soft Landing Using a Tilt-Rotor Mechanism. IEEE Access 2019, 7, 4868-4879. [CrossRef]

28. Kawasaki, K.; Motegi, Y.; Zhao, M.; Okada, K.; Inaba, M. Dual connected bi-copter with new wall trace locomotion feasibility that can fly at arbitrary tilt angle. In Proceedings of the 2015 IEEE/RSJ International Conference on Intelligent Robots and Systems (IROS), Hamburg, Germany, 28 September-2 October 2015; pp. 524-531.

29. Honnery, D. Introduction to the Theory of Flight; Gracie Press: Northcote, Australia, 2000; pp. 94-122.

30. Xiong, L.; Yu, Z.; Wang, Y.; Yang, C.; Meng, Y. Vehicle dynamics control of four in-wheel motor drive electric vehicle using gain scheduling based on tyre cornering stiffness estimation. Veh. Syst. Dyn. 2012, 50, 831-846. [CrossRef] 